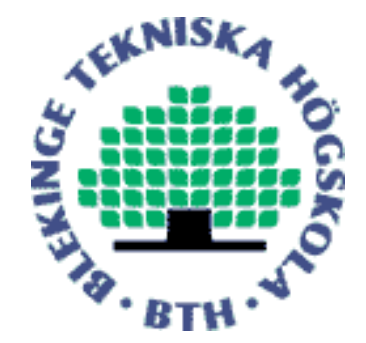

Copyright (C) 2014 IEEE.

Citation for the published paper:

Performance of Cognitive Radio Networks with Finite Buffer Using Multiple Vacations and Exhaustive Service

Hoc Phan, Thi My Chinh Chu, Hans-Jürgen Zepernick, Hien Quoc Ngo

IEEE International Conference on Signal Processing and Communication Systems

2014 Gold Coast, Australia

This material is posted here with permission of the IEEE. Such permission of the IEEE does not in any way imply IEEE endorsement of any of BTH's products or services Internal or personal use of this material is permitted. However, permission to reprint/republish this material for advertising or promotional purposes or for creating new collective works for resale or redistribution must be obtained from the IEEE by sending a blank email message to pubs-permissions@iee.org.

By choosing to view this document, you agree to all provisions of the copyright laws protecting it. 


\title{
Performance of Cognitive Radio Networks with Finite Buffer Using Multiple Vacations and Exhaustive Service
}

\author{
Hoc Phan ${ }^{\dagger, *}$, Thi My Chinh Chu ${ }^{\dagger}$, Hans-Jürgen Zepernick ${ }^{\dagger}$, and Hien Quoc Ngo ${ }^{\ddagger}$ \\ ${ }^{\dagger}$ Blekinge Institute of Technology, Karlskrona, Sweden, E-mail: \{thi.my.chinh.chu, hans-jurgen.zepernick\}@bth.se \\ ${ }^{*}$ University of Reading, RG6 6AY, UK, E-mail: h.phan@ reading.ac.uk \\ ${ }^{\ddagger}$ Linköping University, 58183 Linköping,Sweden, E-mail: nqhien@isy.liu.se
}

\begin{abstract}
In this paper, we analyze the performance of a cognitive radio network where the secondary transmitter, besides its own transmission, occasionally relays the primary signal. It is assumed that the secondary transmitter employs the exhaustive service mode to transmit the secondary signal and multiple vacations to relay the primary signal. When assisting the primary transmitter, we assume that the secondary transmitter utilizes the decode-and-forward protocol to process the primary signal and forwards it to the primary receiver. Furthermore, the secondary transmitter has a finite buffer, the arriving packets of the secondary network are modeled as a Poisson process, and all channels are subject to Nakagami- $m$ fading. Modeling the system as an $M / G / 1 / K$ queueing system with exhaustive service and multiple vacations, using an embedded Markov chain approach to analyze the system, we obtain several key queueing performance indicators, i.e., the channel utilization, blocking probability, mean number of packets, and mean serving time of a packet in the system. The derived formulas are then utilized to evaluate the performance of the considered system.
\end{abstract}

\section{INTRODUCTION}

Due to the growth of ubiquitous wireless communications, which inflicts increasing stress on the limited radio spectrum, cognitive radio technology has been developed as a means of alleviating the scarcity of radio spectrum [1]. In principle, there exist three significant schemes of the spectrum access, i.e., interweave, overlay, and underlay schemes [2]. In the interweave spectrum access, the secondary users opportunistically occupy the licensed spectrum when the primary user does not use its spectrum. This scheme requires the secondary users to sense the status of the primary channels to decide whether or not the channels are idle. In the overlay spectrum access scheme, secondary users can operate on the primary users' spectrum in concurrence with the primary users provided that the secondary network applies interference cancelation techniques to get rid of the interference to the primary network. With underlay spectrum access, a secondary user is allowed to access the primary radio spectrum at any time provided that the interference power at the primary receiver imposed by the secondary transmission is kept below a predefined threshold. The underlay spectrum access is endowed with several important advantages such as high spectrum utilization and simple implementation which has led to an increasing interest in this approach (see [3]-[6], and the references therein).
Although numerous recent studies have investigated outage probability or symbol error rate (SER) for cognitive radio networks (CRNs) (see [7], [8], and the references therein), there are few works evaluating queueing performance such as throughput, transmission delay, channel utilization, and blocking probability for CRNs. Specifically, modeled as an M/G/1 queueing system with infinite buffer, Poisson arrival process, and deterministic departure process, the system performance of a spectrum sharing system has been addressed in [9]. In addition, in [10], considering the Poisson arrival process and deterministic departure process, the performance of an opportunistic spectrum access system, which is modeled as an M/D/1 infinite queue, was investigated. Taking the timeout transmission and infinite queueing buffer into account, the studies of [9], [10] have investigated the queueing performance of a CRN. Recently, assuming a finite buffer at the secondary transmitter, the queueing performance of a $\mathrm{CRN}$, which is modeled as an $\mathrm{M} / \mathrm{G} / 1 / \mathrm{K}$ queueing system, has been assessed in [11].

In this paper, we analyze the queueing behavior of an underlay CRN where the secondary transmitter serves in two roles, transmitting its own signal and relaying the primary signal. The idea of letting the secondary user forward the primary signal has been shown as a means of providing significant spatial diversity for the primary user [12], [13]. In the considered system, we assume that the secondary transmitter is subject to the interference power threshold of the primary receiver. Also, the secondary transmitter is equipped with a finite buffer, its arriving packets follow a Poisson process, and all channels are modeled as Nakagami- $m$ fading. Applying an embedded Markov chain, the secondary system is modeled as an $\mathrm{M} / \mathrm{G} / 1 / \mathrm{K}$ queueing system with multiple vacations and exhaustive service. Then, the blocking probability, channel utilization, mean serving time of packet in the system, and mean number of packets in the system are analyzed.

The rest of this paper is organized as follows. A description of the system and channel models are given in Section II. Section III presents the queueing performance analysis. Selected numerical examples and discussions are provided in Section IV. Finally, Section V concludes the paper.

Notation: The probability density function (PDF) and the cumulative distribution function (CDF) of a random variable 


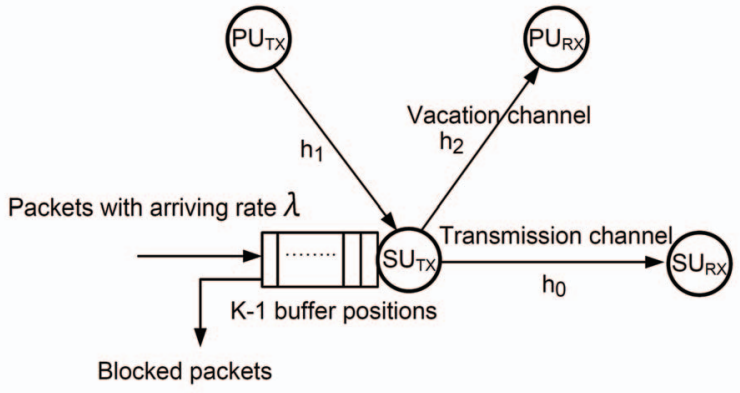

Fig. 1. System model for primary relaying assisted by a secondary network with finite buffer using multiple vacations and exhaustive service.

(RV) $X$ are denoted as $f_{X}(\cdot)$ and $F_{X}(\cdot)$, respectively. $\Gamma(n)$ denotes the gamma function [14, eq. (8.310.1)] and $\Gamma(n, x)$ is the incomplete gamma function [14, eq. (8.350.2)]. Finally, $\mathbb{E}[\cdot]$ is the expectation operator.

\section{SyStem AND ChANNEL MOdel}

We consider a single hop underlay CRN, comprising a secondary transmitter $\mathrm{SU}_{\mathrm{TX}}$ and a secondary receiver $\mathrm{SU}_{\mathrm{RX}}$, coexisting with a primary transmitter $\mathrm{PU}_{\mathrm{TX}}$ and a primary receiver $\mathrm{PU}_{\mathrm{RX}}$ (see Fig. 1). Besides its own transmission, to improve the performance of the primary communication, the secondary transmitter $\mathrm{SU}_{\mathrm{TX}}$ offers to relay the transmission of $\mathrm{PU}_{\mathrm{TX}}$ as long as it has no data in the buffer to transmit. In particular, the secondary transmitter $\mathrm{SU}_{\mathrm{TX}}$ starts a vacation to forward a signal of the primary transmission only if the buffer becomes empty, so-called exhaustive service. We assume that $\mathrm{SU}_{\mathrm{TX}}$ utilizes the decode-and-forward (DF) protocol to relay the primary signal since it can cancel the noise at $\mathrm{SU}_{\mathrm{TX}}$. It continues being on vacation if no secondary packets exist at $\mathrm{SU}_{\mathrm{TX}}$ at the end of each vacation. Until there is at least one packet waiting upon an ended vacation, $\mathrm{SU}_{\mathrm{TX}}$ returns to transmit its own packets. This paradigm is referred to as the multiple vacation model. Usually, the primary transmission is performed through the direct transmission from $\mathrm{PU}_{\mathrm{TX}}$ to $\mathrm{PU}_{\mathrm{RX}}$. However, when $\mathrm{SU}_{\mathrm{TX}}$ goes on vacation, it informs the $\mathrm{PU}_{\mathrm{TX}}$ being available to relay the primary signal. The works of [12], [13] have revealed that with the assistance of $\mathrm{SU}_{\mathrm{TX}}$ in forwarding the primary signal, the performance of the primary transmission is significantly enhanced. Thus, we do not delve into analyzing the performance of the primary network any more. Instead, we focus on analyzing the performance of the secondary network. Assume that all the involved channels are modeled as Nakagami- $m$ fading with fading severity parameter $m$ which covers a wide range of other fading channels as special cases. Let $s_{0}$ be the transmit signal of the secondary network with average transmit power, $P_{0}=\mathbb{E}\left[\left|s_{0}\right|^{2}\right]$. Constrained by the interference power threshold $Q$ imposed by $\mathrm{PU}_{\mathrm{RX}}$ and the transmit power limit, $P_{\max }$, the average transmit power $P_{0}$ of the secondary transmitter is determined as

$$
P_{0}=\min \left\{P_{\max }, Q /\left|h_{2}\right|^{2}\right\}
$$

where $h_{2}$ is the channel coefficient of the link from $\mathrm{SU}_{\mathrm{TX}}$ to $\mathrm{PU}_{\mathrm{RX}}$ with channel mean power $\Omega_{2}$, and positive integer fading severity parameter $m_{2}$. Denote $n_{0}$ as the additive white Gaussian noise (AWGN) at $\mathrm{SU}_{\mathrm{RX}}$ with zero-mean and variance $N_{0}$ and $h_{0}$ as the channel coefficient of the link from $\mathrm{SU}_{\mathrm{TX}}$ to $\mathrm{SU}_{\mathrm{RX}}$ with channel mean power $\Omega_{0}$, and positive integer fading severity parameter $m_{0}$. Then, the received signal at $\mathrm{SU}_{\mathrm{RX}}$ is given by

$$
y_{0}=h_{0} s_{0}+n_{0}
$$

From (1) and (2), the instantaneous signal-to-noise ratio (SNR) of the secondary transmission can be expressed as

$$
\gamma_{0}=\min \left\{\gamma_{\max }\left|h_{0}\right|^{2}, \gamma_{Q}\left|h_{0}\right|^{2} /\left|h_{2}\right|^{2}\right\}
$$

where $\gamma_{\max }=P_{\max } / N_{0}$ and $\gamma_{Q}=Q / N_{0}$. When the secondary user $\mathrm{SU}_{\mathrm{TX}}$ is on vacation to forward the primary signal, the received signal at $\mathrm{PU}_{\mathrm{RX}}$ can be formulated as

$$
y_{2}=h_{2} s_{2}+n_{2}
$$

where $s_{2}$ is the decoded symbol for the primary transmission at $\mathrm{SU}_{\mathrm{TX}}$ which is then forwarded with power $P_{\max }=\mathbb{E}\left[\left|s_{2}\right|^{2}\right]$, and $n_{2}$ is the AWGN at $\mathrm{PU}_{\mathrm{RX}}$ with zero-mean and variance $N_{0}$. Accordingly, the instantaneous SNR of the vacation transmission can be written as

$$
\gamma_{2}=P_{\max }\left|h_{2}\right|^{2} / N_{0}
$$

\section{Performance Analysis of the Secondary NETWORK}

In this section, we analyze the queueing performance of the secondary transmission provided that the secondary transmitter takes vacations for relaying the primary transmission whenever it has no data for its own transmission.

\section{A. Statistical Distributions of Transmission and Vacation Times}

Assume that the packets in the buffer of $\mathrm{SU}_{\mathrm{TX}}$ are transmitted according to the first come first serve (FCFS) principle. The transmission rate of these packets is equal to the channel capacity of the secondary channel from $\mathrm{SU}_{\mathrm{TX}}$ to $\mathrm{SU}_{\mathrm{RX}}$. Thus, a distribution of the transmission rate, which is expressed as a general RV, can be straightforwardly derived from the distribution of the packet transmission time $T_{0}$ over the channel from $\mathrm{SU}_{\mathrm{TX}}$ to $\mathrm{SU}_{\mathrm{RX}}$. This distribution, in turn, depends on the distribution of the corresponding instantaneous SNR according to the Shannon capacity theorem as

$$
T_{0}=\frac{1}{C_{0}}=\frac{1}{b \log _{2}\left(1+\gamma_{0}\right)}[\text { secs } / \text { packet }]
$$

where $b=B / L, B$ is the channel bandwidth in $\mathrm{Hz}$, and $L$ is the packet length in bits. From (6), we can write the CDF of $T_{0}$ as

$$
F_{T_{0}}(t)=1-F_{\gamma_{0}}\left(2^{1 /(b t)}-1\right)
$$

Applying the order statistics theory along with the total probability theorem to (3), we can derive an expression for 
the CDF of the instantaneous SNR $\gamma_{0}$ as

$$
\begin{aligned}
F_{\gamma_{0}}(\gamma) & =1-\sum_{i=0}^{m_{0}-1} \frac{\alpha_{0}^{i} \gamma^{i} e^{-\alpha_{0} \gamma / \gamma_{\max }}}{i ! \gamma_{\max }^{i}}+\frac{\alpha_{0}^{m_{0}}}{\Gamma\left(m_{0}\right)} \sum_{i=0}^{m_{2}-1} \frac{\alpha_{2}^{i} \gamma_{Q}^{i}}{i !} \\
& \times \frac{\gamma^{m_{0}} \Gamma\left(m_{0}+i, \frac{\gamma_{Q} \alpha_{2}+\alpha_{0} \gamma}{\gamma_{\max }}\right)}{\left(\gamma_{Q} \alpha_{2}+\alpha_{0} \gamma\right)^{m_{0}+i}}
\end{aligned}
$$

where $\alpha_{0}=m_{0} / \Omega_{0}$ and $\alpha_{2}=m_{2} / \Omega_{2}$. Substituting (8) into (7), it follows that

$$
\begin{aligned}
& F_{T_{0}}(t)=\sum_{i=0}^{m_{0}-1} \frac{\alpha_{0}^{i}}{i ! \gamma_{\max }^{i}}\left[2^{1 /(b t)}-1\right]^{i} e^{-\alpha_{0}\left[2^{1 /(b t)}-1\right] / \gamma_{\max }} \\
& -\frac{\alpha_{0}^{m_{0}}}{\Gamma\left(m_{0}\right)} \sum_{i=0}^{m_{2}-1} \frac{\alpha_{2}^{i} \gamma_{Q}^{i}}{i !} \frac{\left[2^{1 /(b t)}-1\right]^{m_{0}}}{\left\{\gamma_{Q} \alpha_{2}+\alpha_{0}\left[2^{1 /(b t)}-1\right]\right\}^{m_{0}+i}} \\
& \times \Gamma\left(m_{0}+i, \frac{\gamma_{Q} \alpha_{2}+\alpha_{0}\left[2^{1 /(b t)}-1\right]}{\gamma_{\max }}\right)
\end{aligned}
$$

In principle, packet transmission time can approach positive infinity when the channel quality becomes very poor. Practically, if the packet transmission time goes beyond a predefined threshold, say time-out $t_{o}$, the packet is treated as dropped and the transmission of the subsequent packet follows. The dropping probability that a packet is considered being timed out is determined as, $P_{0}^{o}=\mathrm{P}\left[T_{0} \geq t_{o}\right]=1-F_{T_{0}}\left(t_{o}\right)$. Therefore, we introduce a new RV of packet transmission time $T_{0}^{o}$ capturing this transmission situation, namely, which is defined as

$$
T_{0}^{o}= \begin{cases}T_{0}, & 0<T_{0}<t_{o} \\ t_{o}, & T_{0} \geq t_{o}\end{cases}
$$

Then, the PDF of packet transmission time $T_{0}^{o}$, when considering time-out, can be written as

$$
f_{T_{0}^{o}}(t)= \begin{cases}f_{T_{0}}(t), & 0<t<t_{o} \\ P_{0}^{o}, & t=t_{o} \\ 0, & t>t_{o}\end{cases}
$$

Accordingly, the mean packet transmission time $\mathbb{E}\left[T_{0}^{o}\right]$ can be formulated as

$$
\mathbb{E}\left[T_{0}^{o}\right]=\int_{0}^{t_{o}} t f_{T_{0}}(t) d t+t_{o} P_{0}^{o}=t_{o}-\int_{0}^{t_{o}} F_{T_{0}}(t) d t
$$

Substituting (9) into (12), we obtain

$$
\begin{aligned}
& \mathbb{E}\left[T_{0}^{o}\right]=t_{o}-\sum_{i=0}^{m_{0}-1} \frac{\alpha_{0}^{i}}{i ! \gamma_{\max }^{i}} \int_{0}^{t_{o}}\left[2^{1 /(b t)}-1\right]^{i} e^{-\frac{\alpha_{0}\left[2^{1 /(b t)}-1\right]}{\gamma_{\max }}} d t \\
& +\frac{\alpha_{0}^{m_{0}}}{\Gamma\left(m_{0}\right)} \sum_{i=0}^{m_{2}-1} \frac{\alpha_{2}^{i} \gamma_{Q}^{i}}{i !} \int_{0}^{t_{o}} \frac{\left[2^{1 /(b t)}-1\right]^{m_{0}}}{\left\{\gamma_{Q} \alpha_{2}+\alpha_{0}\left[2^{1 /(b t)}-1\right]\right\}^{m_{0}+i}} \\
& \times \Gamma\left(m_{0}+i, \frac{\gamma_{Q} \alpha_{2}}{\gamma_{\max }}+\frac{\alpha_{0}\left[2^{1 /(b t)}-1\right]}{\gamma_{\max }}\right) d t
\end{aligned}
$$

By using a similar derivation for the CDF $F_{T_{0}}(t)$ and the mean packet transmission time $\mathbb{E}\left[T_{0}^{o}\right]$, respectively, we obtain the $\mathrm{CDF}$ of vacation time $T_{2}$ and the mean vacation time $\mathbb{E}\left[T_{2}^{o}\right]$ as

$$
F_{T_{2}}(t)=\sum_{i=0}^{m_{2}-1} \frac{\alpha_{2}^{i}}{i ! \gamma_{\max }^{i}}\left[2^{1 /(b t)}-1\right]^{i} e^{-\frac{\alpha_{2}}{\gamma_{\max }}\left[2^{1 /(b t)}-1\right]}
$$

$$
\mathbb{E}\left[T_{2}^{o}\right]=t_{o}-\sum_{i=0}^{m_{2}-1} \frac{\alpha_{2}^{i}}{i ! \gamma_{\max }^{i}} \int_{0}^{t_{o}}\left[2^{1 /(b t)}-1\right]^{i} e^{-\frac{\alpha_{2}\left[2^{1 /(b t)}-1\right]}{\gamma_{\max }}} d t
$$

\section{B. Queueing Performance of the Secondary Network}

In this section, we further evaluate the performance of the considered secondary network in terms of its queueing behavior. We assume that the maximum number of packets in the secondary system is $K$, including one packet being transmitted and $K-1$ packets waiting in the buffer. Because of the finite buffer length, as long as this buffer is completely occupied, new arriving packets cannot join the system and have to leave it without being transmitted. Assume that secondary packets arriving at $\mathrm{SU}_{\mathrm{TX}}$ can be modeled as a Poisson process with arrival rate $\lambda$. Also, these packets are transmitted with a rate equal to the channel capacity of the secondary channel from $\mathrm{SU}_{\mathrm{TX}}$ to $\mathrm{SU}_{\mathrm{RX}}$. When being on vacation for relaying the primary signal, the primary packets are forwarded with a rate equal to the channel capacity of the link from $\mathrm{SU}_{\mathrm{TX}}$ to $\mathrm{PU}_{\mathrm{RX}}$. Therefore, an $\mathrm{M} / \mathrm{G} / 1 / \mathrm{K}$ queueing system with multiple vacations and exhaustive service can be applied to model the network depicted in Fig. 1. The embedded Markov points of the underlying Markov chain are selected immediately after either a secondary transmission completion or a vacation transmission completion. In addition, each state of the embedded Markov chain at the $n$-th Markov point is identified as a combination of the number of packets, $L_{n}$, in $\mathrm{SU}_{\mathrm{TX}}$ immediately after the $n$-th Markov point and the type of transmission completion $\zeta_{n}$ :

$$
\zeta_{n}= \begin{cases}0, & \text { primary relaying completion } \\ 1, & \text { secondary transmission completion }\end{cases}
$$

Then, the states of the embedded Markov chain is denoted as $L_{n}^{\left(\zeta_{n}\right)}$. For the states that correspond to the embedded Markov points selected immediately after a primary transmission completion $\left(\zeta_{n}=0\right), L_{n}$ can take a value from 0 up to $K$. However, for the states that correspond to the embedded Markov points selected immediately after a secondary transmission completion $\left(\zeta_{n}=1\right), L_{n} \in\{0, \ldots, K-1\}$. Let $\pi_{k}^{(0)}$ and $\pi_{k}^{(1)}$ be the probabilities that $k$ packets exist in the buffer of $\mathrm{SU}_{\mathrm{TX}}$ immediately after a primary transmission completion and immediately after a secondary transmission completion, respectively. Clearly, $\pi_{k}^{(0)}$ and $\pi_{k}^{(1)}$ are the steadystate probabilities of State $k^{(0)}$ and State $k^{(1)}$, respectively. As defined in [15, eq. (3.2)], $\pi_{k}^{(0)}$ and $\pi_{k}^{(1)}$ are, respectively, 
calculated as

$$
\begin{gathered}
\pi_{k}^{(0)}=\lim _{n \rightarrow \infty} \operatorname{Pr}\left[L_{n}^{\left(\zeta_{n}\right)}=k^{(0)}\right]=\lim _{n \rightarrow \infty} \operatorname{Pr}\left[\zeta_{n}=0, L_{n}=k\right] \\
\text { for } 0 \leq k \leq K \\
\pi_{k}^{(1)}=\lim _{n \rightarrow \infty} \operatorname{Pr}\left[L_{n}^{\left(\zeta_{n}\right)}=k^{(1)}\right]=\lim _{n \rightarrow \infty} \operatorname{Pr}\left[\zeta_{n}=1, L_{n}=k\right] \\
\text { for } 0 \leq k \leq K-1
\end{gathered}
$$

The state transition probability $p_{j, k}^{(u v)}$ from State $j^{(u)}$ to State $k^{(v)}$ of the Markov chain is defined as

$$
p_{j, k}^{(u v)}=\operatorname{Pr}\left[\zeta_{n+1}=v, L_{n+1}=k \mid \zeta_{n}=u, L_{n}=j\right]
$$

In particular, these transition probabilities can be classified into the following cases:

- Case 1: The state transition probabilities from State $0^{(0)}$ to State $k^{(0)}$ and from State $0^{(1)}$ to State $k^{(0)}, 0 \leq k \leq$ $K-1$, i.e.,

$$
p_{0, k}^{(00)}=p_{0, k}^{(10)}=f_{k}, 0 \leq k \leq K-1
$$

where $f_{k}$ represents the probability that $k$ packets arrive at $\mathrm{SU}_{\mathrm{TX}}$ during its vacation time for relaying the primary signal and $f_{k}$ can be expressed as [15, eq. (3.6)]

$$
\begin{gathered}
f_{k}=\int_{0}^{t_{o}} \frac{(\lambda t)^{k}}{k !} e^{-\lambda t} f_{T_{2}}(t) d t+\frac{\left(\lambda t_{o}\right)^{k}}{k !} e^{-\lambda t_{o}} P_{2}^{o}=\frac{\left(\lambda t_{o}\right)^{k}}{k !} \\
\times e^{-\lambda t_{o}}+\int_{0}^{t_{o}} F_{T_{2}}(t) \frac{\lambda e^{-\lambda t}\left((\lambda t)^{k}-k(\lambda t)^{k-1}\right)}{k !} d t
\end{gathered}
$$

Substituting (14) into (21) gives

$$
\begin{aligned}
& f_{k}=\frac{\left(\lambda t_{o}\right)^{k}}{k !} e^{-\lambda t_{o}}+\sum_{i=0}^{m_{2}-1} \frac{\alpha_{2}^{i}}{i ! \gamma_{\max }^{i}} \int_{0}^{t_{o}}\left[2^{1 /(b t)}-1\right]^{i} \\
& \times e^{-\frac{\alpha_{2}}{\gamma_{\max }}\left[2^{1 /(b t)}-1\right]} \frac{\lambda e^{-\lambda t}\left((\lambda t)^{k}-k(\lambda t)^{k-1}\right)}{k !} d t
\end{aligned}
$$

- Case 2: The state transition probabilities from State $0^{(0)}$ to State $K^{(0)}$ and from State $0^{(1)}$ to State $K^{(0)}$ are

$$
p_{0, K}^{(00)}=p_{0, K}^{(10)}=1-\sum_{k=0}^{K-1} f_{k}
$$

- Case 3: The state transition probabilities from State $j^{(0)}$ to State $k^{(1)}$ and from State $j^{(1)}$ to State $k^{(1)}, 0 \leq k \leq$ $K-2,1 \leq j \leq k+1$, are given by

$p_{j, k}^{(01)}=p_{j, k}^{(11)}=a_{k-j+1}, 0 \leq k \leq K-2,1 \leq j \leq k+1$

where $a_{k}$ is the probability that $k$ packets arrive at $\mathrm{SU}_{\mathrm{TX}}$ during a secondary transmission time, i.e.,

$$
\begin{aligned}
& a_{k}=\int_{0}^{t_{o}} \frac{(\lambda t)^{k}}{k !} e^{-\lambda t} f_{T_{0}}(t) d t+\frac{\left(\lambda t_{o}\right)^{k}}{k !} e^{-\lambda t_{o}} P_{0}^{o} \\
& =\frac{\left(\lambda t_{o}\right)^{k} e^{-\lambda t_{o}}}{k !}+\int_{0}^{t_{o}} F_{T_{0}}(t) \frac{\lambda e^{-\lambda t}\left((\lambda t)^{k}-k(\lambda t)^{k-1}\right)}{k !} d t
\end{aligned}
$$

Substituting (9) in (25), we have

$$
\begin{aligned}
& a_{k}=\frac{\left(\lambda t_{o}\right)^{k}}{k !} e^{-\lambda t_{o}}+\sum_{i=0}^{m_{0}-1} \frac{\alpha_{0}^{i}}{i ! \gamma_{\max }^{i}} \int_{0}^{t_{o}}\left[2^{1 /(b t)}-1\right]^{i} \\
& \times e^{-\frac{\alpha_{0}}{\gamma_{\max }}\left[2^{1 /(b t)}-1\right]} \frac{\lambda e^{-\lambda t}\left[(\lambda t)^{k}-k(\lambda t)^{k-1}\right]}{k !} d t \\
& -\frac{\alpha_{0}^{m_{0}}}{\Gamma\left(m_{0}\right)} \sum_{i=0}^{m_{2}-1} \frac{\alpha_{2}^{i} \gamma_{Q}^{i}}{i !} \int_{0}^{t_{o}} \frac{\Gamma\left(m_{0}+i, \frac{\gamma_{Q} \alpha_{2}}{\gamma_{\max }}+\frac{\alpha_{0}\left[2^{1 /(b t)}-1\right]}{\gamma_{\max }}\right)}{\left(\gamma_{Q} \alpha_{2}+\alpha_{0}\left[2^{1 /(b t)}-1\right]\right)^{m_{0}+i}} \\
& \times\left[2^{1 /(b t)}-1\right]^{m_{0}} \frac{\lambda e^{-\lambda t}\left((\lambda t)^{k}-k(\lambda t)^{k-1}\right)}{k !} d t
\end{aligned}
$$

- Case 4: The state transition probabilities from State $j^{(0)}$ to State $(K-1)^{(1)}$, from State $j^{(1)}$ to State $(K-1)^{(1)}$, and from State $K^{(0)}$ to State $(K-1)^{(1)}, 1 \leq j \leq K-1$, are

$$
\begin{aligned}
& p_{j, K-1}^{(01)}=p_{j, K-1}^{(11)}=\sum_{k=K-j}^{\infty} a_{k}=1-\sum_{k=0}^{K-j-1} a_{k}, \\
& p_{K, K-1}^{(01)}=1
\end{aligned}
$$

Therefore, the set of limiting state probability equations and the probability conservation equation are given by

$$
\begin{aligned}
& \pi_{k}^{(0)}=\pi_{0}^{(0)} p_{0, k}^{(00)}+\pi_{0}^{(1)} p_{0, k}^{(10)}=\left(\pi_{0}^{(0)}+\pi_{0}^{(1)}\right) f_{k}, \\
& 0 \leq k \leq K-1 \\
& \pi_{K}^{(0)}=\pi_{0}^{(0)} p_{0, K}^{(00)}+\pi_{0}^{(1)} p_{0, K}^{(10)}=\left(\pi_{0}^{(0)}+\pi_{0}^{(1)}\right)\left(1-\sum_{k=0}^{K-1} f_{k}\right)
\end{aligned}
$$

$$
\pi_{k}^{(1)}=\sum_{j=1}^{k+1}\left[\pi_{j}^{(0)} p_{j, k}^{(01)}+\pi_{j}^{(1)} p_{j, k}^{(11)}\right]=\sum_{j=1}^{k+1}\left(\pi_{j}^{(0)}+\pi_{j}^{(1)}\right) a_{k-j+1},
$$$$
0 \leq k \leq K-2
$$

$\pi_{K-1}^{(1)}=\sum_{j=1}^{K-1}\left[\pi_{j}^{(0)} p_{j, K-1}^{(01)}+\pi_{j}^{(1)} p_{j, K-1}^{(11)}\right]+\pi_{K}^{(0)}$

$$
\sum_{k=0}^{K} \pi_{k}^{(0)}+\sum_{k=0}^{K-1} \pi_{k}^{(1)}=1
$$

In order to find $\pi_{k}^{(0)}, k \in(0, \ldots, K)$, and $\pi_{k}^{(1)}, \quad k \in$ $(0, \ldots, K-1)$, we only need $2 K+1$ independent equations. From (28), (29), (30), and (32), we can express the limiting state probability equations in matrix form as

$$
\mathbf{a}=\boldsymbol{\pi} \mathbf{P}
$$

where $\mathbf{a}$ is a $1 \times(2 K+1)$ vector, defined as

$$
\mathbf{a}=\left(\begin{array}{lllllll}
0 & 0 & 0 & \ldots & 0 & 0 & 1
\end{array}\right)
$$

The limiting state probabilities are stacked into the vector $\pi$ as

$$
\boldsymbol{\pi}=\left(\pi_{0}^{(0)} \pi_{1}^{(0)} \ldots \pi_{K}^{(0)} \pi_{0}^{(1)} \pi_{1}^{(1)} \ldots \pi_{K-1}^{(1)}\right)
$$




$$
\mathbf{P}=\left(\begin{array}{cccccccccccccc}
f_{0}-1 & f_{1} & f_{2} & \ldots & f_{K-2} & f_{K-1} & 1-\sum_{k=0}^{K-1} f_{k} & 0 & 0 & 0 & \ldots & 0 & 0 & 1 \\
0 & -1 & 0 & \ldots & 0 & 0 & 0 & a_{0} & a_{1} & a_{2} & \ldots & a_{K-3} & a_{K-2} & 1 \\
0 & 0 & -1 & \ldots & \vdots & \vdots & \vdots & 0 & a_{0} & a_{1} & \ldots & a_{K-4} & a_{K-3} & 1 \\
\vdots & \vdots & 0 & \ddots & 0 & 0 & 0 & \vdots & 0 & a_{0} & \ddots & \vdots & \vdots & 1 \\
0 & 0 & \vdots & \ldots & -1 & 0 & 0 & 0 & \vdots & 0 & \ldots & a_{0} & a_{1} & 1 \\
0 & 0 & 0 & \ldots & 0 & -1 & 0 & 0 & 0 & \vdots & \ldots & 0 & a_{0} & 1 \\
0 & 0 & 0 & \ldots & 0 & 0 & -1 & 0 & 0 & 0 & \ldots & 0 & 0 & 1 \\
f_{0} & f_{1} & f_{2} & \ldots & f_{K-2} & f_{K-1} & 1-\sum_{k=0}^{K-1} f_{k} & -1 & 0 & 0 & \ldots & 0 & 0 & 1 \\
0 & 0 & 0 & \ldots & 0 & 0 & 0 & a_{0} & a_{1}-1 & a_{2} & \ldots & a_{K-3} & a_{K-2} & 1 \\
0 & 0 & 0 & \ldots & 0 & 0 & 0 & 0 & a_{0} & a_{1}-1 & \ldots & a_{K-4} & a_{K-3} & 1 \\
\vdots & \vdots & \vdots & \ddots & \vdots & \vdots & \vdots & \vdots & 0 & a_{0} & \ddots & \vdots & \vdots & 1 \\
0 & 0 & 0 & \ldots & 0 & 0 & 0 & 0 & \vdots & 0 & \ldots & a_{1}-1 & \vdots & 1 \\
0 & 0 & 0 & \ldots & 0 & 0 & 0 & 0 & 0 & \vdots & \ldots & a_{0} & a_{1}-1 & 1 \\
0 & 0 & 0 & \ldots & 0 & 0 & 0 & 0 & 0 & 0 & \ldots & 0 & a_{0} & 1
\end{array}\right)
$$

Eventually, $\mathbf{P}$ is an $(2 K+1) \times(2 K+1)$ matrix determined as (36). As a result, the linear equation system in (33) gives the solution to the limiting state probabilities as

$$
\boldsymbol{\pi}=\mathbf{a} \mathbf{P}^{-1}
$$

\section{Channel Utilization}

The channel utilization $\rho^{\prime}$, defined as the long-run fraction of time that the secondary channel is utilized, is given by $[15$, eq. (3.12a)]

$$
\rho^{\prime}=\frac{\left(1-\pi_{0}^{(0)}-\pi_{0}^{(1)}\right) \mathbb{E}\left[T_{0}^{o}\right]}{\left(\pi_{0}^{(0)}+\pi_{0}^{(1)}\right) \mathbb{E}\left[T_{2}^{o}\right]+\left(1-\pi_{0}^{(0)}-\pi_{0}^{(1)}\right) \mathbb{E}\left[T_{0}^{o}\right]}
$$

\section{Blocking Probability}

Blocking probability is defined as the probability that an arriving packet is not accepted to the system when it is totally full. By definition, the blocking probability for the considered system is given by [15, eq. (1.20b)]

$$
P_{B}=1-\frac{\rho^{\prime}}{\rho}
$$

where the offered load $\rho$ is calculated as

$$
\rho=\lambda \mathbb{E}\left[T_{0}^{o}\right]
$$

Substituting (38) and (40) into (39), we get

$$
P_{B}=1-\frac{1}{\lambda} \frac{\left(1-\pi_{0}^{(0)}-\pi_{0}^{(1)}\right)}{\left(\pi_{0}^{(0)}+\pi_{0}^{(1)}\right) \mathbb{E}\left[T_{2}^{o}\right]+\left(1-\pi_{0}^{(0)}-\pi_{0}^{(1)}\right) \mathbb{E}\left[T_{0}^{o}\right]}
$$

\section{E. Mean Number of Packets in the System}

Mean number of packets in the secondary system is expressed as [15, eq. (3.31)]

$$
\begin{aligned}
& \mathbb{E}[L]=\frac{\sum_{k=1}^{K-1} k \pi_{k}^{(1)}}{\lambda\left[\left(\pi_{0}^{(0)}+\pi_{0}^{(1)}\right) \mathbb{E}\left[T_{2}^{o}\right]+\left(1-\pi_{0}^{(0)}-\pi_{0}^{(1)}\right) \mathbb{E}\left[T_{0}^{o}\right]\right]} \\
& +K P_{B}
\end{aligned}
$$

\section{F. Mean Serving Time of a Packet in the System}

Mean serving time of a packet in the secondary system is defined as the sum of the waiting time of a packet in the buffer of $\mathrm{SU}_{\mathrm{TX}}$ and its transmission time. For the considered system, the mean time in the system can be computed as [15, eq. (3.69a)]

$$
\begin{aligned}
& \mathbb{E}[T]=\frac{\mathbb{E}\left[T_{0}^{o}\right] \sum_{k=1}^{K-1} k \pi_{k}^{(1)}}{\lambda \rho^{\prime}\left[\left(\pi_{0}^{(0)}+\pi_{0}^{(1)}\right) \mathbb{E}\left[T_{2}^{o}\right]+\left(1-\pi_{0}^{(0)}-\pi_{0}^{(1)}\right) \mathbb{E}\left[T_{0}^{o}\right]\right]} \\
& +\frac{K\left(\rho-\rho^{\prime}\right)}{\lambda \rho^{\prime}}
\end{aligned}
$$

\section{Numerical Results AND Discussion}

In this section, we present numerical examples to illustrate the queueing performance of a secondary system wherein the secondary transmitter assists the primary transmission by using multiple vacations and exhaustive service. In all scenarios, the time-out threshold, bandwidth, and the number of bits per packet are, respectively, selected as $t_{o}=100 \mathrm{~ms}, B=1 \mathrm{MHz}$, and $N=4096$. In addition, we select the average SNR as $\gamma_{\max }=P_{\max } / N_{0}=15 \mathrm{~dB}$. Assume that all the channel mean powers are attenuated according to the exponential decaying model wherein the path-loss exponent is chosen as $\nu=4$ representing a highly shadowed urban area. The normalized distances from $\mathrm{SU}_{\mathrm{TX}}$ to $\mathrm{SU}_{\mathrm{RX}}$ and from $\mathrm{SU}_{\mathrm{TX}}$ to $\mathrm{PU}_{\mathrm{RX}}$ are $d_{0}=2$ and $d_{2}=4$. In addition, the fading severity parameters 


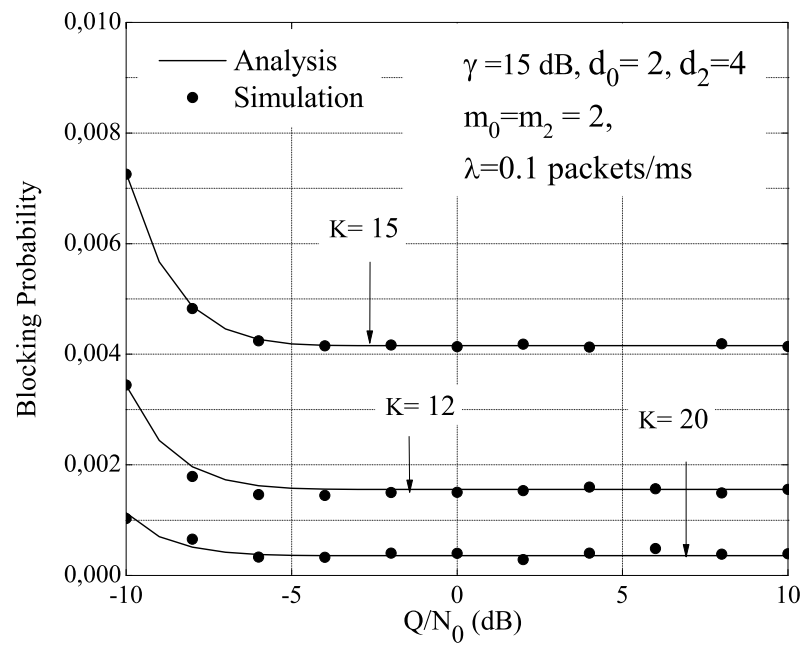

Fig. 2. Blocking probability against interference power-to-noise ratio $Q / N_{0}$ for various buffer lengths $K$.

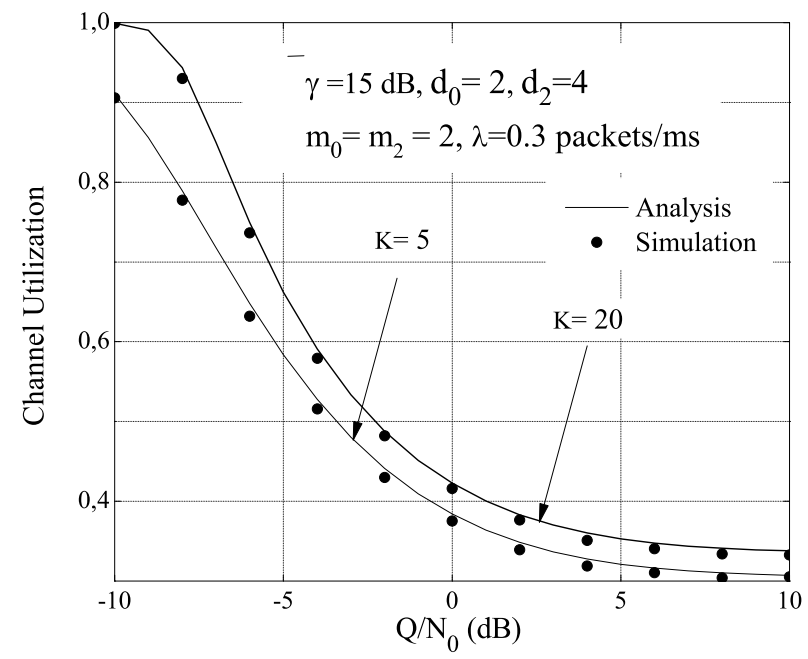

Fig. 3. Channel utilization against interference power-to-noise ratio $Q / N_{0}$ for various buffer lengths $K$.

of the links from $\mathrm{SU}_{\mathrm{TX}}$ to $\mathrm{SU}_{\mathrm{RX}}$ and from $\mathrm{SU}_{\mathrm{TX}}$ to $\mathrm{PU}_{\mathrm{RX}}$ are $m_{0}=2$ and $m_{2}=2$.

Fig. 2 shows the blocking probability against interference power-to-noise ratio $Q / N_{0}$ for various buffer lengths $K$. As can be observed from this figure, when the buffer length becomes large, the blocking probability decreases. This implies that arriving packets have higher probability to be accepted to the system for transmission. Furthermore, at low value of $Q / N_{0}$, the blocking probability decreases with the increasing of $Q / N_{0}$. However, when the tolerable interference powerto-noise ratio increases beyond a threshold, the blocking probability remains constant. This effect is attributed to the fact that in the high interference power-to-noise ratio regime, the transmit power of $\mathrm{SU}_{\mathrm{TX}}$ is limited to $P_{\max }$, according to (1).

Fig. 3 plots channel utilization against interference power-

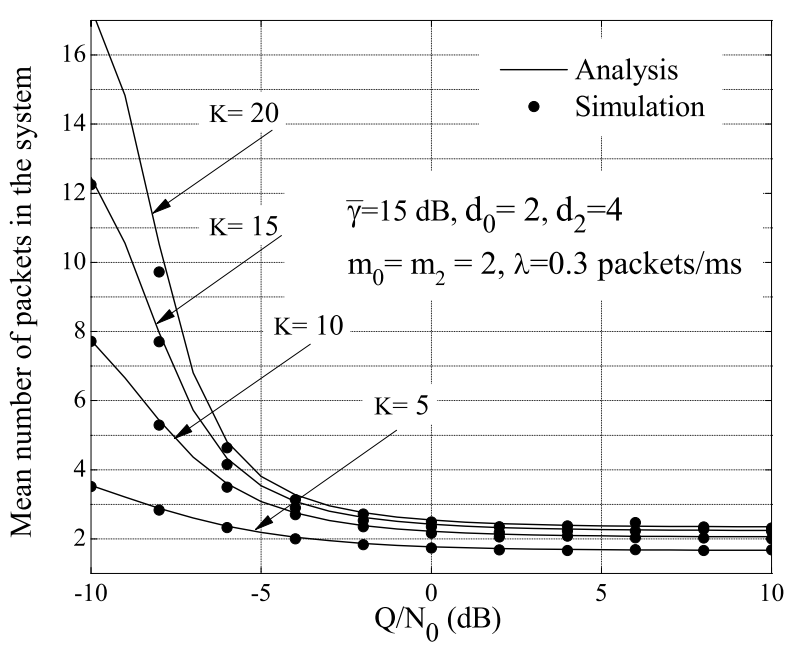

Fig. 4. Mean number of packets in the system against interference powerto-noise ratio $Q / N_{0}$ for various buffer lengths $K$.

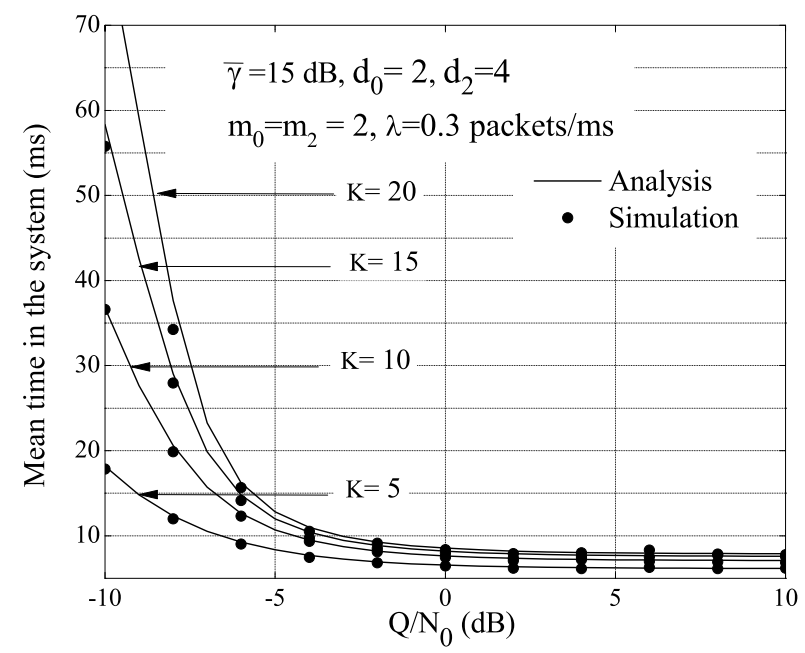

Fig. 5. Mean time in the system against interference power-to-noise ratio $Q / N_{0}$ for various buffer lengths $K$.

to-noise ratio $Q / N_{0}$ for various buffer lengths $K$. As expected, the channel utilization increases according to the increase of the buffer length. Accordingly, when the buffer length becomes large there are more arriving packets to be accepted to the system and, therefore, more packets will be transmitted. When the interference power-to-noise ratio $Q / N_{0}$ becomes sufficiently large, the channel utilization becomes saturated. This effect is because in the high regime of the interference power-to-noise ratio, the transmit power of $\mathrm{SU}_{\mathrm{TX}}$ is equal to the transmit power limit $P_{\max }$ that leads to the saturated channel utilization.

Fig. 4 shows the mean number of packets in the system against interference power-to-noise ratio $Q / N_{0}$ for various buffer lengths $K$. As can be seen from Fig. 4, the mean number of packets in the system becomes large when the buffer length increases. In addition, at high values of $Q / N_{0}$, the transmit 
power of $\mathrm{SU}_{\mathrm{TX}}$ is only constrained by the transmit power limit $P_{\max }$, such that there exists a floor of the mean number of packets in the system.

Fig. 5 shows the mean time in the system against interference power-to-noise ratio $Q / N_{0}$ for various buffer lengths $K$. For the low regime of $Q / N_{0}$, i.e., from $-10 \mathrm{~dB}$ to $0 \mathrm{~dB}$, we can see that increasing the buffer length $K$ results in an increase of the mean time in the system. This is because when the buffer length increases, the mean number of packets in the system also increases. As a result, the mean time that a packet spends in the system will increase. However, for higher values of $Q / N_{0}$, as the buffer length $K$ varies, the mean time in the system changes slightly. This is because the transmission rate becomes very large such that the secondary transmitter can serve all the waiting packets immediately. Thus, the effect of the buffer length $K$ on the packet waiting time in the buffer becomes insignificant.

\section{Conclusions}

We have analyzed the performance of a secondary cognitive system over Nakagami- $m$ fading where the secondary transmitter is on vacation to forward the primary signal. We have considered the scenario where the secondary transmitter adopts multiple vacations and exhaustive service. Also, the secondary transmitter has a finite buffer and its arriving packets are modeled as a Poisson process. Utilizing an embedded Markov chain to analyze the corresponding M/G/1/K queueing system with multiple vacations and exhaustive service, we have derived several key queueing performance indicators for the examined network. In particular, blocking probability, channel utilization, mean number of packets in the system, and mean time in the system of the secondary network are evaluated. Finally, selected numerical examples are presented to investigate the impact of various network parameters on the performance of the examined secondary network.

\section{REFERENCES}

[1] J. Mitola, "Cognitive radio: An integrated agent architecture for software defined radio," Ph.D. dissertation, Royal Inst. of Technology, Stockholm, Sweden, 2000.

[2] S. Srinivasa and S. Jafar, "Cognitive radios for dynamic spectrum access - the throughput potential of cognitive radio: A theoretical perspective," IEEE Commun. Mag., vol. 45, no. 5, pp. 73-79, May 2007.

[3] A. Ghasemi and E. S. Sousa, "Fundamental limits of spectrum-sharing in fading environments," IEEE Trans. Wireless Commun., vol. 6, no. 2, pp. 649-658, Feb. 2007.

[4] L. Musavian and S. Aïssa, "Ergodic and outage capacities of spectrum sharing systems in fading channels," in Proc. IEEE Global Telecommunications Conference, Washington, DC, USA, Nov. 2007, pp. 3327-3331.

[5] S. Haykin, "Cognitive radio: Brain-empowered wireless communications," IEEE J. Sel. Areas Commun., vol. 23, no. 2, pp. 201-220, Feb. 2005.

[6] A. Goldsmith, S. A. Jafar, I. Maric, and S. Srinivasa, "Breaking spectrum gridlock with cognitive radios: An information theoretic perspective," Proceedings of the IEEE, vol. 97, no. 5, pp. 894-914, May 2009.

[7] Y. Zou, Y.-D. Yao, and B. Zheng, "Outage probability analysis of cognitive transmissions: Impact of spectrum sensing overhead," IEEE Trans. Wireless Commun., vol. 9, no. 8, pp. 2676-2688, Aug. 2010

[8] L. Liang, P. I. Derwin, and M. Pesavento, "Symbol error rate analysis in multiuser underlay cognitive radio systems," in Proc. IEEE International Symposium on Personal, Indoor and Mobile Radio Communications, Toronto, Canada, Sep. 2011, pp. 681-684.
[9] A. K. Farraj, S. L. Miller, and K. A. Qaraqe, "Queue performance measures for cognitive radios in spectrum sharing systems," in Proc. IEEE Global Telecommunications Conference Workshops, Houston, Texas, USA, Dec. 2011, pp. 997-1001.

[10] I. Suliman and J. Lehtomaki, "Queueing analysis of opportunistic access in cognitive radios," in Proc. Second International Workshop on Cognitive Radio and Advanced Spectrum Management, Aalborg, Denmark, May 2009, pp. 153-157.

[11] T. M. C. Chu, H. Phan, and H.-J. Zepernick, "On the performance of underlay cognitive radio networks using M/G/1/K queueing model," IEEE Communications Letters, vol. 17, no. 5, pp. 876-879, May 2013.

[12] W. Jaafar, W. Ajib, and D. Haccoun, "A novel relay-aided transmission scheme in cognitive radio networks," in Proc. IEEE Global Telecommunications Conference, Houston, Texas, USA, Dec. 2011, pp. 1-6.

[13] T. M. C. Chu, H. Phan, and H.-J. Zepernick, "Amplify-and-forward relay assisting both primary and secondary transmissions in cognitive radio networks over Nakagami- $m$ fading," in Proc. IEEE International Symposium on Personal, Indoor and Mobile Radio Communications, Sydney, Australia, Sep. 2012, pp. 932- 937.

[14] I. S. Gradshteyn and I. M. Ryzhik, Table of Integrals, Series and Products, 6th ed. A. Jeffrey, Ed. Academic Press, 2000.

[15] H. Takagi, Queueing Analysis: A Foundation of Performance Evaluation. Vol. 2. Finite Systems. Netherlands: Elsevier, 1993. 